\title{
ALTERAÇÕES EM ALGUMAS PROPRIEDADES DE UM LATOSSOLO DEGRADADO COM USO DE LODO DE ESGOTO E RESÍDUOS ORGÂNICOS ${ }^{(1)}$
}

\author{
Priscila Torres Modesto(2), Márcia Helena Scabora ${ }^{(3)}$, Gilberto \\ Colodro $^{(4)}$, Katia Luciene Maltoni ${ }^{(5)}$ \& Ana Maria Rodrigues \\ Cassiolato $^{(5)}$
}

\begin{abstract}
RESUMO
A recuperação de áreas muito impactadas, como as oriundas da construção de hidrelétricas, é um processo lento e, usualmente, requer a adição de resíduos orgânicos, como fonte de matéria orgânica, e de nutrientes, como condicionador das propriedades do solo. O objetivo deste trabalho foi verificar o efeito da adição de lodo de esgoto e resíduos orgânicos (maravalha e torta de filtro de cana-deaçúcar) sobre o crescimento de duas espécies arbóreas de Cerrado na recuperação de um subsolo de uma área degradada pela construção da Usina Hidrelétrica de Ilha Solteira, localizada em Selvíria - MS. O experimento foi conduzido em casa de vegetação em delineamento experimental inteiramente casualizado, com oito tratamentos, seis repetições e uma planta por repetição, para cada planta-teste. Cada repetição (saco plástico) teve $3,3 \mathrm{~L}$ e as proporções em volume da mistura de cada resíduo foram de: $30 \%$ de lodo de esgoto, $20 \%$ de torta de filtro e $10 \%$ de maravalha de madeira. Foram mensuradas a fertilidade, a micorrização, a atividade microbiana ( $\mathrm{C}-\mathrm{CO}_{2}$ liberado) do subsolo, a massa do sistema radicular e parte aérea e a altura de plantas de duas espécies nativas do Cerrado [monjoleiro (Acacia polyphylla DC.) e jatobá-do-Cerrado (Hymenaea stigonocarpa Mart)]. Os tratamentos com lodo de esgoto proporcionaram maior atividade microbiana no substrato e maior crescimento para as duas espécies arbóreas. A presença do lodo de esgoto promoveu melhora na fertilidade do subsolo, com aumento dos teores de matéria orgânica, $\mathrm{P}, \mathrm{K}$, Ca e Mg. A matéria orgânica e o $\mathrm{P}$ tiveram seus teores elevados nos tratamentos com mistura de lodo de esgoto e demais resíduos. Os
\end{abstract}

\footnotetext{
(1) Parte do trabalho de graduação do primeiro autor. Recebido para publicação em maio de 2006 e aprovado em maio de 2009.

(2) Graduada em Ciências Biológicas, Universidade Federal de Mato Groso do Sul - UFMS. Av. Ranulpho Marques Leal, 3.484 Caixa Postal 210, CEP 79620-080 Três Lagoas (MS).

(3) Doutoranda em Agronomia, Universidade Estadual Paulista - UNESP. Campus de Ilha Solteira. Caixa Postal 31, Av. Brasil 51, CEP 15385-000 Ilha Solteira (SP). E-mail: scaboramh@yahoo.com.br

${ }^{(4)}$ Centro Federal de Educação Tecnológica - CEFET de Rio Verde (GO). Rod. Sulgoiana, km 1, s/n, CEP 75901-970 Rio Verde (GO). E-mail: gilbertocolodro@bol.com.br

${ }^{(5)}$ Professora Assistente do Departamento de Fitossanidade, Engenharia Rural e Solos - UNESP. E-mails: malatoni@agr.feis.unesp.br; anamaria@bio.feis.unesp.br
} 
resultados permitem concluir que as misturas contendo lodo associado a resíduos promoveram melhores incrementos na qualidade do subsolo, com perspectivas de sua recuperação.

Termos de indexação: torta de filtro, maravalha, atividade microbiana, micorriza arbuscular, recuperação de áreas degradadas.

\section{SUMMARY: ALTERATIONS IN SOME PROPERTIES OF A DEGRADED OXISOL BY SEWAGE SLUDGE AND ORGANIC RESIDUE APPLICATION}

The recovery of intensively impacted areas, as originated by the construction of a hydroelectric power plant, is a slow process and usually requires the addition of organic residues as source of organic matter and nutrients to improve the soil properties. The objective of this study was to verify the effects of the addition of sewage sludge and organic residues (wood shavings and sugar cane filter cake) on the growth of two Cerrado tree species, used in the recovery of the properties of a subsoil area impacted by the construction of the hydroelectric power plant Ilha Solteira in the county of Selviria, MS, Brazil. The experiment was conducted in a greenhouse and the experimental design consisted of randomized blocks with eight treatments, six replications with one plant per replication, for each test plant. Each replication (a 3.3 L plastic bag) was conducted with the following mixture proportions: $30 \%$ sewage sludge; $20 \%$ filter cake, and $10 \%$ wood shavings. Subsoil fertility, mycorrhizal colonization, microbial activity (released $\mathrm{C}-\mathrm{CO}_{2}$ ), and plant growth (canopy and root fresh and dry matter weight and plant heights 30, 60, 90 and 120 days after planting) of two Cerrado native species ['monjoleiro'(Acacia polyphylla DC.) and 'jatobá-do-cerrado'(Hymenaea stigonocarpa Mart)] were analyzed as indicators of subsoil recovery. The treatments with sewage sludge resulted in increased microbial activity in the substrate and increased growth of the two tree species. The presence of sewage sludge improved subsoil fertility, increasing the organic matter, $P, K, C a$ and $M g$ contents. The levels of organic matter and $P$ were elevated in the treatments with sewage mixture and the other residues, indicating such applications as adequate in recovery processes. The results showed that the mixtures containing sewage associated to residues improved the subsoil quality with a view to its recovery.

Index terms: filter cake, wood shavings, microbial activity, arbuscular micorriza, recovery of degraded areas.

\section{INTRODUÇÃO}

Com o grande crescimento populacional, surgiu a necessidade da ocupação dos Cerrados para fins de produção agrícola (Fernandes \& Muraoka, 2002), em virtude de sua localização estratégica e das características físicas dos solos, que facilitam a mecanização. Entretanto, a degradação tem sido observada em decorrência de manejos inadequados, oriundos de atividades antrópicas (construção de estradas, hidrelétricas, atividades de mineração, etc.), especialmente as praticadas nas áreas agrícolas. O denominador comum dessas áreas degradadas é a remoção do horizonte superficial juntamente com a matéria orgânica, causando sérios problemas físicos, químicos e biológicos ao solo (Duda et al., 1999), dando origem às ‘áreas de empréstimo'.

A recuperação de áreas degradadas é um processo lento e, como tal, composto por várias etapas, que devem ser realizadas em sequência, visando restabelecer o seu potencial de produção (Santos et al., 2001). Uma das alternativas para o destino de resíduos orgânicos pode ser sua incorporação ao solo, que, servindo como meio depurador, poderia elevar a produtividade de culturas agrícolas por meio da melhoria da fertilidade, com maior ou menor intensidade, dependendo das características do resíduo adicionado. Esse tipo de resíduo, por vezes, poderia ser muito importante na recuperação de áreas degradadas com reflorestamento, cujos produtos não são utilizados na alimentação humana.

A torta de filtro de cana-de-açúcar, subproduto da agroindústria de açúcar e álcool, é considerada um excelente fertilizante, além de propiciar melhores condições para germinação das culturas. Aumentos da produção levaram Prasad (1976) a concluir ser desnecessária a utilização de $\mathrm{P}$ quando se aplica torta de filtro em dose superior a $20 \mathrm{t} \mathrm{ha}^{-1}$ no sulco de plantio.

No Brasil, um sério problema ambiental é a contaminação dos solos e dos lençóis freáticos pelo 
acúmulo de resíduos das indústrias madeireiras, como serragem, cascas, capilhos e outros. Em serrarias da região Sul, cerca de $14 \%$ do volume de toras processadas transforma-se em serragem. Embora parte desse volume seja usada como combustível ou componente da indústria de chapas, existe um grande excedente, principalmente em pequenas serrarias, nas quais o rendimento no processamento é geralmente menor. Usualmente, parte dessa serragem é queimada ou disposta em aterros inadequados, acarretando o desprendimento de chorumes de alta carga orgânica tóxica, o que provoca danos aos mananciais. A utilização da serragem como condicionador de solos é limitada pela lentidão com que esse material é degradado em condições naturais, devido à estabilidade de seus componentes, como a lignina e a celulose (Branco et al., 2005).

A microbiota do solo, em face de seu papel na ciclagem de nutrientes, é importante na reconstrução da fertilidade e fornece um indicativo precoce de mudanças no conteúdo de matéria orgânica, sendo sua estimativa, por meio do $\mathrm{CO}_{2}$ liberado, uma análise de baixo custo e alta eficiência, indicada como ferramenta para avaliar a recuperação de áreas degradadas, sinalizando mudanças rapidamente (M. Neto \& Souza, 2000). A medição da respiração é uma forma de estimar a atividade dos microrganismos do solo e um indicativo da velocidade de decomposição da matéria orgânica ou de materiais a ele adicionados (Severino et al., 2004).

Outros microrganismos, como os fungos micorrízicos arbusculares (FMA), podem também ser empregados como indicadores biológicos da qualidade e do equilíbrio de um sistema (Moreira \& Siqueira, 2006). Essas associações mutualistas, por meio da colonização radicular e da produção de micélio externo, podem atingir sítios fora da zona de depleção de nutrientes, aumentando a absorção e o aproveitamento da água e dos nutrientes do solo (Caldeira et al., 1997).

Espécies arbóreas como o jatobá-do-Cerrado ou jatobá-do-campo (Hymenaea stigonocarpa Mart.) e monjoleiro (Acacia polyphylla DC.) são características de formações abertas do Cerrado e Campo-Cerrado. O jatobá-do-Cerrado é muito procurado pela fauna, sendo, por isso, útil nos plantios em áreas degradadas destinadas à recomposição da vegetação arbórea. $\mathrm{O}$ monjoleiro, uma leguminosa que ocorre com frequência nos Estados de Mato Grosso do Sul, São Paulo e Paraná, apresenta porte médio, crescimento rápido nos estádios iniciais da sucessão secundária e potencial para uso na recuperação de áreas degradadas.

Considerando esses aspectos das espécies vegetais, a importância da atividade microbiológica na decomposição de resíduos orgânicos, o desenvolvimento de associações mutualistas que podem favorecer o desenvolvimento de espécies vegetais e a necessidade de dispor de resíduos orgânicos adequadamente e de recuperar áreas degradadas, foi desenvolvido o presente trabalho, que teve por objetivo verificar o efeito da adição do lodo de esgoto e resíduos orgânicos (maravalha de madeira e torta de filtro de cana-deaçúcar) sobre o crescimento de duas espécies arbóreas de Cerrado e na recuperação de um subsolo de uma área degradada pela construção da Usina Hidrelétrica de Ilha Solteira.

\section{MATERIAL E MÉTODOS}

O experimento foi conduzido em casa de vegetação na Faculdade de Engenharia da UNESP Universidade Estadual Paulista/campus de Ilha Solteira. O material de solo utilizado, doravante denominado subsolo exposto, é proveniente de uma "área de empréstimo", localizada no município de Selvíria, MS, nas coordenadas de $22^{\circ} 22^{\prime} \mathrm{S}$ e $51^{\circ} 24^{\prime} \mathrm{W}$. O solo predominante na área foi classificado por Demattê (1980) como Latossolo Vermelho. Originalmente, a região apresentava como cobertura vegetal o Cerrado sensu stricto (Rodrigues et al., 2007). No final da década de 1960, com a construção da Usina Hidrelétrica de Ilha Solteira, o local foi desmatado e uma parte dessa área foi utilizada como 'área de empréstimo', de onde o solo foi retirado até a profundidade de $8 \mathrm{~m}$. Atualmente, neste local, pequenas áreas estão em processo de regeneração natural, mas, em sua maior extensão o subsolo permanece exposto.

As espécies vegetais selecionadas como indicadoras foram o monjoleiro (Acacia polyphylla DC.) e jatobádo-Cerrado (Hymenaea stigonocarpa Mart), cujas sementes foram tratadas superficialmente com hipoclorito de sódio $2 \mathrm{cL} \mathrm{L}^{-1}$, lavadas com água destilada e colocadas para germinar em gerbox. As plântulas mais sadias foram transferidas para os sacos plásticos contendo os substratos. No transplante, foram adicionados $50 \mathrm{~g}$ de solo de uma área de Cerrado preservado como reinoculante de microrganismos.

Os resíduos orgânicos utilizados foram a maravalha, obtida em uma serraria localizada na cidade de Selvíria (MS), a torta de filtro de cana-deaçúcar, proveniente da Destivale - Destilaria Vale do Tietê S/A, e o lodo de esgoto, obtido na SANEAR Saneamento de Araçatuba S/A, ambos do município de Araçatuba, SP. A análise do lodo (Quadro 1) mostrou teores equivalentes a 57,12 e $70 \mathrm{~g} \mathrm{~kg}^{-1} \mathrm{de} \mathrm{N}$, $\mathrm{P}_{2} \mathrm{O}_{5}$ e $\mathrm{K}_{2} \mathrm{O}$, respectivamente. Em relação aos metais pesados, o lodo apresentou valores inferiores aos limites estabelecidos pelas principais agências de fiscalização ambiental, tendo em vista sua origem de efluentes domésticos (Colodro et al., 2007). A composição microbiológica média desse tipo de lodo foi de 0,04 ovos $\mathrm{g}^{-1}$ de sólidos totais tanto de protozoários como de helmintos, valores abaixo do limite máximo determinado pela CETESB (1999), que é de 0,25 ovos $\mathrm{g}^{-1}$ de sólidos totais, sendo, dessa forma, classificado como lodo do tipo B, sem restrições para uso agrícola. 
Quadro 1. Características químicas e físicas dos resíduos e limites máximos permitidos de alguns elementos-traço para o lodo de esgoto

\begin{tabular}{|c|c|c|c|c|}
\hline \multirow{2}{*}{ Característica $^{(1)}$} & \multirow{2}{*}{ Unidade $^{(2)}$} & \multicolumn{3}{|c|}{ Teores e características } \\
\hline & & Lodo & Torta & Maravalha \\
\hline \multicolumn{5}{|c|}{ Físicas } \\
\hline $\mathrm{pH}$ (in natura) & & 7,1 & 5,2 & -(4) \\
\hline Umidade & dag kg-1 & 85,0 & 70,0 & 10,0 \\
\hline Sólidos voláteis & dag $\mathrm{kg}^{-1}$ & 71,5 & & \\
\hline Carbono orgânico & $\mathrm{g} \mathrm{kg}^{-1}$ & 406,0 & 300,0 & 490,0 \\
\hline Relação $\mathrm{C} / \mathrm{N}$ & & $7: 1$ & $23: 1$ & $140: 1$ \\
\hline \multicolumn{5}{|c|}{ Macronutrientes } \\
\hline $\mathrm{N}$ amoniacal & $\mathrm{g} \mathrm{kg}^{-1}$ & 8,9 & - & 1,0 \\
\hline N nitrato-nitrito & $\mathrm{g} \mathrm{kg}^{-1}$ & 105,7 & & 0,2 \\
\hline $\mathrm{N}$ total & $\mathrm{g} \mathrm{kg}^{-1}$ & 57,1 & 13,0 & 3,5 \\
\hline Fósforo & $\mathrm{g} \mathrm{kg}^{-1}$ & 27,7 & 4,8 & 0,2 \\
\hline Potássio & $\mathrm{g} \mathrm{kg}^{-1}$ & 8,4 & 6,3 & 0,8 \\
\hline Cálcio & $\mathrm{g} \mathrm{kg}^{-1}$ & 3,9 & 7,0 & 1.8 \\
\hline Magnésio & $\mathrm{g} \mathrm{kg}^{-1}$ & 3,3 & 1,6 & 0,4 \\
\hline Enxofre & $\mathrm{g} \mathrm{kg}^{-1}$ & 3,0 & - & 0,1 \\
\hline \multicolumn{5}{|c|}{ Micronutrientes e $\mathrm{Al}$} \\
\hline Boro & $\mathrm{mg} \mathrm{kg}^{-1}$ & 10,7 & - & 26,0 \\
\hline Cobre & $\mathrm{mg} \mathrm{kg}^{-1}$ & 159,8 & - & 18,0 \\
\hline Ferro & $\mathrm{mg} \mathrm{kg}^{-1}$ & $7.385,0$ & - & 177,0 \\
\hline Manganês & $\mathrm{mg} \mathrm{kg}^{-1}$ & 77,8 & - & 132,0 \\
\hline Zinco & $\mathrm{mg} \mathrm{kg}^{-1}$ & 474,4 & - & 48,0 \\
\hline Molibdênio & $\mathrm{mg} \mathrm{kg}^{-1}$ & ND & - & - \\
\hline \multirow[t]{3}{*}{ Alumínio } & $\mathrm{mg} \mathrm{kg}^{-1}$ & $4.968,0$ & - & - \\
\hline & \multicolumn{4}{|c|}{ Elementos-traço do lodo de esgoto } \\
\hline & & & CETESB & US-EPA \\
\hline Arsênio & $\mathrm{mg} \mathrm{kg}^{-1}$ & $\mathrm{ND}^{(3)}$ & 75,0 & 41,0 \\
\hline Cádmio & $\mathrm{mg} \mathrm{kg}^{-1}$ & 1,6 & 85,0 & 39,0 \\
\hline Chumbo & $\mathrm{mg} \mathrm{kg}^{-1}$ & 28,7 & 840,0 & 300,0 \\
\hline Cromo total & $\mathrm{mg} \mathrm{kg}^{-1}$ & 20,4 & - & $1.200,0$ \\
\hline Mercúrio & $\mathrm{mg} \mathrm{kg}^{-1}$ & ND & 57,0 & 17,0 \\
\hline Níquel & $\mathrm{mg} \mathrm{kg}^{-1}$ & 18,1 & 420 & 420 \\
\hline Selênio & $\mathrm{mg} \mathrm{kg}^{-1}$ & ND & 100 & 36 \\
\hline Sódio & $\mathrm{mg} \mathrm{kg}^{-1}$ & $1.255,0$ & & \\
\hline
\end{tabular}

(1) Método empregado para metais SW3051, USEPA, determinação por ICP-AES; ${ }^{(2)}$ Valores de concentração com base na matéria seca e o de sólidos voláteis, com relação ao total de sólidos. CETESB: Companhia de Saneamento do Estado de São Paulo (CETESB, 1999). US-EPA: Norma 40 CFR Part 503 (USEPA, 1993) com limites para lodo de qualidade excepcional. ${ }^{(3)} \mathrm{ND}$ : Não detectado. ${ }^{(4)}$ Não analisado.

$\mathrm{O}$ delineamento experimental foi o inteiramente casualizado, em esquema fatorial $2 \times 3$, com oito tratamentos e seis repetições. Cada planta foi utilizada como uma repetição, para cada uma das duas espécies vegetais (monjoleiro e jatobá-do-Cerrado). Cada repetição (saco plástico) teve capacidade para 3,3 L de substrato, e os tratamentos consistiram de: T1: subsolo exposto + inóculo + calcário (controle); T2: controle + lodo de esgoto; T3: controle + lodo de esgoto+ torta de filtro; T4: controle + lodo de esgoto + maravalha de madeira; T5: controle + lodo de esgoto + torta de filtro + maravalha de madeira; T6: controle + maravalha de madeira; T7: controle + torta de filtro; e T8: controle + torta de filtro + maravalha de madeira.
A quantidade de resíduos misturada ao subsolo exposto foi determinada a partir da proporção entre eles, sendo de 30,20 e $10 \%$ para lodo de esgoto, torta de filtro e maravalha de madeira à base úmida, respectivamente. Considerando-se a umidade de 85 , 70 e $10 \mathrm{dag} \mathrm{kg}^{-1}$, foram misturados ao volume de $3,3 \mathrm{~L}$ de subsolo exposto $0,15,0,20$ e $0,30 \mathrm{~L}$ de lodo, torta e maravalha, à base seca, respectivamente. Transformando essas proporções em massa, considerando a densidade de 1,0,1,0 e $0,34 \mathrm{~kg} \mathrm{dm}^{-3}$, foram misturados $0,15,0,20$ e $0,10 \mathrm{~kg}$ de lodo, torta $\mathrm{e}$ maravalha ao volume de subsolo, respectivamente (proporção de 1,5:2:1). Para reduzir a compactação e permitir melhor desenvolvimento do sistema radicular, foi adicionada areia grossa ao solo na proporção de 3:1 (subsolo exposto: areia). Os sacos plásticos (unidades experimentais) foram mantidos em casa de vegetação e com rega frequente, por 210 dias, antes da introdução das plântulas, para possibilitar a decomposição inicial do material orgânico, não interferindo no desenvolvimento das plântulas. Todos os tratamentos sofreram correção da acidez do subsolo exposto com aplicação de 6,25 g de calcário dolomítico, por unidade experimental, com base na análise de fertilidade da área de origem do subsolo exposto.

Após esse período, as plântulas foram introduzidas nas unidades experimentais, e o experimento foi conduzido por 120 dias (de outubro/2004 a fevereiro/ 2005). A altura das plantas foi avaliada a cada 30 dias com régua e, após 120 dias, a parte aérea foi cortada e pesada para verificação da produção de matéria fresca; posteriormente, ela foi levada à estufa para secagem a $60^{\circ} \mathrm{C}$ e pesada para determinação do peso da matéria seca. O sistema radicular foi separado do substrato, lavado, verificado quanto ao comprimento e de matéria da massa fresca e, posteriormente, levado à estufa para secagem a $60^{\circ} \mathrm{C}$, para determinação do peso da matéria seca. As raízes foram separadas do substrato com auxílio de peneira de $2 \mathrm{~mm}$ de malha. Em cada repetição, 1,0 g de raiz fina foi separada e preservada em álcool $50 \%$, para avaliação da colonização micorrízica. A colonização micorrízica foi verificada em placa quadriculada, em que 100 segmentos foram avaliados por repetição, sob microscópio estereoscópico (40x), segundo método proposto por Phillips \& Hayman (1970).

O substrato foi peneirado (malha de $2 \mathrm{~mm}$ ) e homogeneizado, sendo efetuadas análises químicas no Laboratório de Fertilidade do Solo da UNESP/campus de Ilha Solteira, onde foram determinados o $\mathrm{pH}$ $\left(\mathrm{CaCl}_{2}\right)$, matéria orgânica, $\mathrm{P}, \mathrm{K}^{+}, \mathrm{Ca}^{2+}, \mathrm{Mg}^{2+}, \mathrm{H}+\mathrm{Al}$, CTC e V, de acordo com os métodos descritos por Raij \& Quaggio (1983).

Parte do substrato foi separada em amostras de $100 \mathrm{~g}$ por repetição, sendo empregada para a quantificação da respiração basal microbiana $\left(\mathrm{C}-\mathrm{CO}_{2}\right.$ liberado) (Anderson \& Domsch, 1982) e para quantificação do número de esporos de FMA. Nessa análise, os esporos foram separados e coletados por 
decantação e peneiramento úmido (Gerdemann \& Nicolson, 1963) e centrifugação e flutuação com sacarose (Jenkins, 1964). A contagem dos esporos foi realizada com microscópio estereoscópico (40x).

Para cada uma das variáveis realizou-se a análise de variância, seguida das comparações de médias por meio do teste de Scott-Knott. Avaliaram-se, também, os coeficientes de correlação linear entre os caracteres.

\section{RESULTADOS E DISCUSSÃO}

As características químicas do substrato, para os diferentes tratamentos e plantas, estão apresentadas no quadro 2. Os valores de $\mathrm{pH}$ mais elevados foram observados no controle e no tratamento com maravalha para monjoleiro e para jatobá-do-Cerrado. A elevação do $\mathrm{pH}$ a valores maiores que 5,5, em todos os tratamentos, promoveu a precipitação do $\mathrm{Al}$ (Catan \& Alonso, 1969).

Com relação ao $\mathrm{P}$, observou-se aumento significativo nos tratamentos com lodo de esgoto em virtude da presença de elevados teores desse elemento
(Quadro 1). Considerando uma taxa de mineralização desse elemento de $70 \%$ para o primeiro ano de cultivo (Raij et al., 1997), foram disponibilizados cerca de 3,0 g em $3,3 \mathrm{dm}^{3}$ de solo, o que equivale a uma dose de $4,16 \mathrm{t} \mathrm{ha}^{-1}$ de $\mathrm{P}_{2} \mathrm{O}_{5}$. Entre os tratamentos que receberam lodo de esgoto, observou-se redução no teor de $\mathrm{P}$ quando associado aos outros resíduos. Provavelmente essa redução tenha ocorrido devido ao menor teor de $\mathrm{P}$ contido na torta (equivalente a $\left.600 \mathrm{~kg} \mathrm{ha}^{-1}\right)$ e na maravalha $\left(12 \mathrm{~kg} \mathrm{ha}^{-1}\right) \mathrm{e}$, também, ao aumento da população de microrganismos do solo, que foi observado em decorrência do aumento da atividade microbiana no tratamento com torta de filtro e maravalha (Quadro 3).

Aumentos significativos nos teores de matéria orgânica foram encontrados nos tratamentos com mistura de lodo e demais resíduos (Quadro 2). No tratamento com lodo aplicado isoladamente o teor de matéria orgânica foi maior apenas que o do controle (subsolo exposto + calagem), mas menor que dos tratamentos em que outros resíduos foram adicionados, para as duas espécies vegetais. A associação de lodo, torta e maravalha foi a que produziu maior elevação no teor de matéria orgânica, o que, provavelmente, ocorreu em razão dos elevados teores de $\mathrm{C}$ desses resíduos, principalmente da maravalha (Quadro 1)

Quadro 2. Características químicas do solo para os diferentes substratos sob monjoleiro e jatobá-do-Cerrado. Média de seis repetições

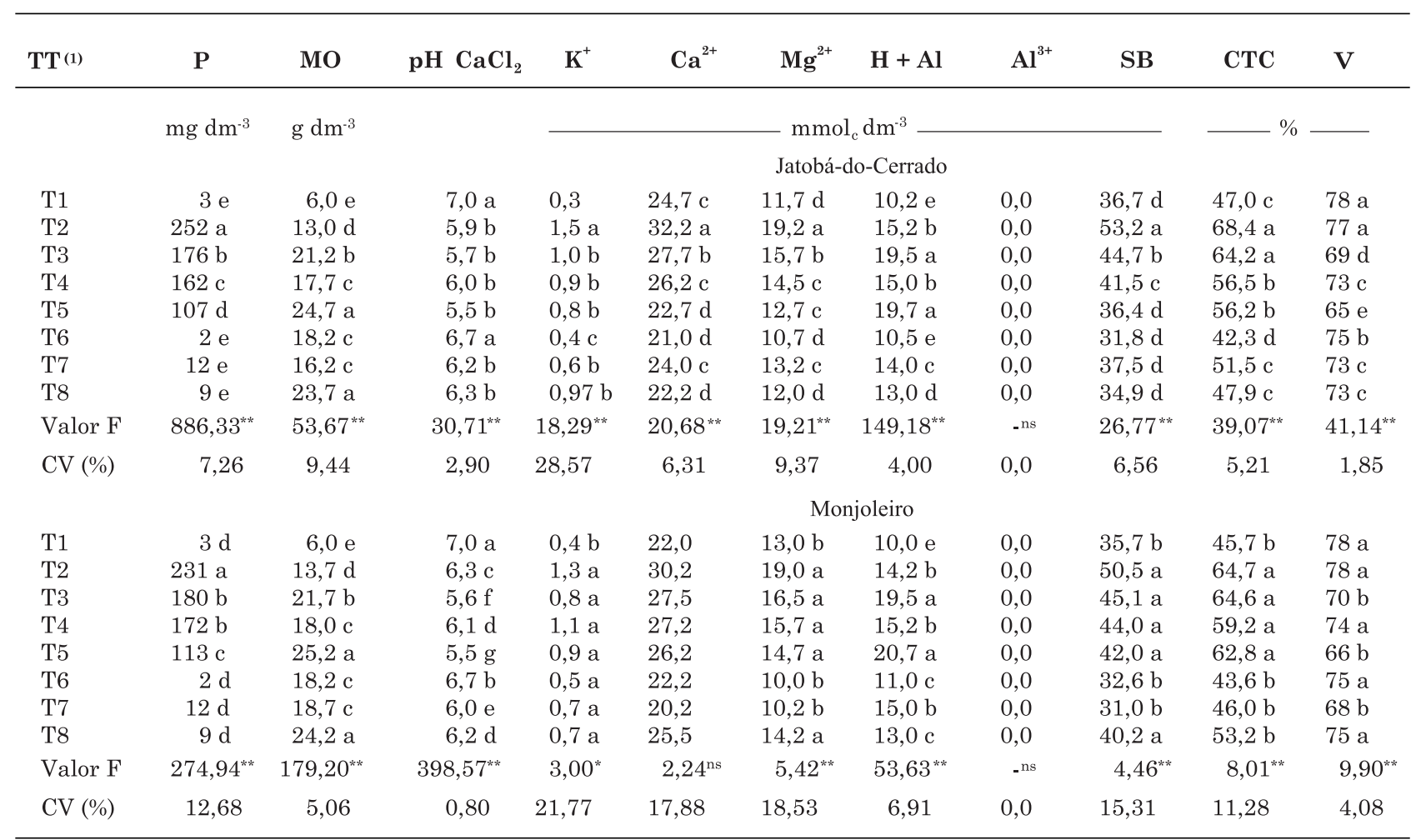

(1) Tratamentos (T): 1. subsolo exposto + inóculo + calcário (controle); 2. controle + lodo de esgoto $(\mathrm{L}) ; 3$. controle $+\mathrm{L}+$ torta de filtro (T); 4. controle $+\mathrm{L}+$ maravalha de madeira $(\mathrm{M}) ; 5$. controle $+\mathrm{L}+\mathrm{T}+\mathrm{M}$; 6. controle $+\mathrm{M}$; 7 . controle $+\mathrm{T}$; e 8 . controle $+\mathrm{T}+\mathrm{M}$. Médias seguidas de mesma letra, na coluna, não diferem entre si pelo teste de Scott-Knott a 5 \%. ${ }^{(2)} \mathrm{CV}$ : coeficiente de variação (\%). 
Quadro 3. Peso da matéria seca da parte aérea (MSPA), de matéria fresca da parte aérea (MFPA), de matéria seca do sistema radicular (MSSR), de matéria fresca do sistema radicular (MFSR), comprimento do sistema radicular (CSR), atividade microbiana do solo (C-CO liberado - $\mathrm{mg} \mathrm{CO}_{2} \mathrm{~g}^{-1}$ solo seco d $\mathrm{d}^{-1}$ ), taxa de colonização micorrízica (COL) e número de esporos de fungos micorrízicos arbusculares (n. esporos FMA por 100 g solo seco) para as plantas de jatobá-do-Cerrado e monjoleiro, nos diferentes substratos. Média de seis repetições

\begin{tabular}{|c|c|c|c|c|c|c|c|c|}
\hline Tratamento $^{(1)}$ & MSPA & MFPA & MSSR & MFSR & CSR & $\begin{array}{c}\mathrm{C}-\mathrm{CO}_{2} \\
\text { liberado }\end{array}$ & COL & $\begin{array}{c}\text { n. }{ }^{(3)} \\
\text { esporos }\end{array}$ \\
\hline & \multicolumn{5}{|c|}{$-g$} & \multirow{2}{*}{\multicolumn{3}{|c|}{$\%$}} \\
\hline & & & & Jatobá- & rrado & & & \\
\hline $\mathrm{T} 1$ & $4,26 \mathrm{c}$ & $9,30 \mathrm{c}$ & $5,66 \mathrm{a}$ & $13,71 \mathrm{c}$ & $29,00 \mathrm{a}$ & $4,27 \mathrm{~d}$ & $66,20 \mathrm{~b}$ & $4,33 \mathrm{~b}$ \\
\hline $\mathrm{T} 2$ & $6,47 \mathrm{~b}$ & $15,38 \mathrm{~b}$ & $3,91 \mathrm{~b}$ & $11,60 \mathrm{c}$ & $26,16 \mathrm{a}$ & $9,56 \mathrm{~b}$ & $26,00 \mathrm{c}$ & $2,50 \mathrm{~b}$ \\
\hline T3 & $10,02 \mathrm{a}$ & $28,72 \mathrm{a}$ & $6,24 \mathrm{a}$ & $16,55 \mathrm{~b}$ & $20,33 \mathrm{~b}$ & $10,70 \mathrm{~b}$ & $76,88 \mathrm{~b}$ & $4,33 \mathrm{~b}$ \\
\hline $\mathrm{T} 4$ & $10,32 \mathrm{a}$ & $26,19 \mathrm{a}$ & $7,02 \mathrm{a}$ & $18,78 \mathrm{a}$ & $22,83 \mathrm{~b}$ & $11,10 \mathrm{~b}$ & $91,16 \mathrm{a}$ & $40,16 \mathrm{a}$ \\
\hline T5 & $10,94 \mathrm{a}$ & $26,96 \mathrm{a}$ & $7,98 \mathrm{a}$ & $21,65 \mathrm{a}$ & $24,33 \mathrm{~b}$ & $13,30 \mathrm{a}$ & $93,16 \mathrm{a}$ & $20,33 \mathrm{a}$ \\
\hline T6 & $3,08 \mathrm{c}$ & $6,29 \mathrm{c}$ & $5,18 \mathrm{~b}$ & $13,71 \mathrm{c}$ & $26,33 \mathrm{a}$ & $6,88 \mathrm{c}$ & $67,83 \mathrm{~b}$ & $1,66 \mathrm{~b}$ \\
\hline $\mathrm{T} 7$ & $4,87 \mathrm{~b}$ & $11,95 \mathrm{~b}$ & $6,66 \mathrm{a}$ & $16,45 \mathrm{~b}$ & $19,50 \mathrm{~b}$ & $6,88 \mathrm{c}$ & $92,16 \mathrm{a}$ & $16,16 \mathrm{a}$ \\
\hline $\mathrm{T} 8$ & $2,71 \mathrm{c}$ & $7,89 \mathrm{c}$ & $3,71 \mathrm{~b}$ & $11,28 \mathrm{c}$ & $29,66 \mathrm{a}$ & $10,44 \mathrm{~b}$ & $99,50 \mathrm{a}$ & $6,16 \mathrm{~b}$ \\
\hline Valor de F & $23,34^{* *}$ & $30,39^{* *}$ & $7,64^{* *}$ & $7,61^{* *}$ & $4,00^{* *}$ & $42,59^{\star * \star}$ & $16,58^{* *}$ & $4,04^{* *}$ \\
\hline \multirow[t]{2}{*}{$\mathrm{CV}(\%)$} & 26,30 & 24,97 & 23,36 & 21,45 & 18,46 & 11,86 & 18,87 & 59,81 \\
\hline & \multicolumn{8}{|c|}{ Monjoleiro } \\
\hline $\mathrm{T} 1$ & $0,21 \mathrm{~d}$ & $0,40 \mathrm{~d}$ & $0,16 \mathrm{c}$ & $0,70 \mathrm{c}$ & $24,33 \mathrm{a}$ & $3,98 \mathrm{e}$ & $1,83 \mathrm{c}$ & $1,66 \mathrm{~b}$ \\
\hline $\mathrm{T} 2$ & $17,64 \mathrm{a}$ & $35,39 \mathrm{a}$ & $4,78 \mathrm{~b}$ & $14,46 \mathrm{~b}$ & $22,50 \mathrm{~b}$ & $12,12 \mathrm{a}$ & $74,80 \mathrm{a}$ & $35,66 \mathrm{a}$ \\
\hline T3 & 17,98 a & $34,85 \mathrm{a}$ & $7,29 \mathrm{a}$ & $22,74 \mathrm{a}$ & $25,50 \mathrm{a}$ & $10,96 \mathrm{~b}$ & $22,83 \mathrm{~b}$ & $6,66 \mathrm{~b}$ \\
\hline $\mathrm{T} 4$ & $9,81 \mathrm{c}$ & $20,53 \mathrm{c}$ & $3,56 \mathrm{~b}$ & $12,38 \mathrm{~b}$ & $25,83 \mathrm{a}$ & $12,64 \mathrm{a}$ & $29,00 \mathrm{~b}$ & $12,33 \mathrm{a}$ \\
\hline T5 & $12,62 \mathrm{~b}$ & $25,81 \mathrm{~b}$ & $4,18 \mathrm{~b}$ & $15,41 \mathrm{~b}$ & $27,16 \mathrm{a}$ & $12,66 \mathrm{a}$ & $76,00 \mathrm{a}$ & $28,16 \mathrm{a}$ \\
\hline $\mathrm{T} 6$ & $0,30 \mathrm{~d}$ & $0,45 \mathrm{~d}$ & $0,21 \mathrm{c}$ & $0,83 \mathrm{c}$ & $18,83 \mathrm{~b}$ & $7,00 \mathrm{c}$ & $16,33 \mathrm{~b}$ & $1,50 \mathrm{~b}$ \\
\hline $\mathrm{T} 7$ & $0,86 \mathrm{~d}$ & $2,43 \mathrm{~d}$ & $0,29 \mathrm{c}$ & $2,02 \mathrm{c}$ & $20,16 \mathrm{~b}$ & $5,83 \mathrm{~d}$ & $28,80 \mathrm{~b}$ & $1,00 \mathrm{~b}$ \\
\hline $\mathrm{T} 8$ & $1,27 \mathrm{~d}$ & $2,46 \mathrm{~d}$ & $0,41 \mathrm{c}$ & $1,37 \mathrm{c}$ & $25,66 \mathrm{a}$ & $11,00 \mathrm{~b}$ & $6,16 \mathrm{c}$ & $4,16 \mathrm{~b}$ \\
\hline Valor de F & $85,39^{* *}$ & $101,35^{* *}$ & $47,01^{* *}$ & $75,29^{\star *}$ & $2,72^{*}$ & $101,45^{* *}$ & $17,34^{* *}$ & $6,61^{* *}$ \\
\hline $\mathrm{CV}(\%)$ & 27,94 & 24,83 & 38,92 & 27,15 & 18,55 & 8,86 & 52,47 & 55,51 \\
\hline
\end{tabular}

(1) Tratamentos (T): 1. subsolo exposto + inóculo + calcário (controle); 2. controle + lodo de esgoto (L); 3. controle + L + torta de filtro $(\mathrm{T}) ; 4$. controle $+\mathrm{L}+$ maravalha de madeira $(\mathrm{M}) ; 5$. controle $+\mathrm{L}+\mathrm{T}+\mathrm{M}$; 6. controle $+\mathrm{M}$; 7 . controle $+\mathrm{T}$; e 8 . controle $+\mathrm{T}$ + M. Médias seguidas de mesma letra, na coluna, não diferem entre si pelo teste de Scott-Knott a $5 \%$. ${ }^{(2)} \mathrm{CV}$ : coeficiente de variação (\%). ${ }^{(3)}$ Opção de transformação: raiz quadrada de $\mathrm{x}+0,5$.

Para as duas espécies arbóreas verificaram-se os maiores teores de $\mathrm{K}$ e $\mathrm{Mg}$ no tratamento contendo apenas lodo (Quadro 2), embora o lodo de esgoto seja considerado um material pobre em $\mathrm{K}$, devido à remoção deste para a porção líquida durante o tratamento do esgoto (Tsutiya, 2001). O tratamento com adição de maravalha, quando não combinado com os demais resíduos orgânicos ricos em nutrientes e de rápida decomposição (como o lodo e a torta de filtro), exibiu os menores teores de Ca, $\mathrm{Mg}$ e CTC para o jatobá-do-Cerrado.

No que diz respeito à altura das plantas, o jatobádo-Cerrado (Quadro 3) não exibiu diferenças estatísticas entre os tratamentos aos 30 dias, porém, nas demais épocas, as maiores alturas foram detectadas para a mistura lodo/maravalha e lodo/ torta/maravalha. Para o monjoleiro, desde os 30 dias, houve diferenças significativas, sendo as maiores alturas encontradas nos tratamentos com lodo e naqueles com associação de resíduos orgânicos. As maiores alturas, verificadas aos 120 dias para as duas espécies, ocorreram no tratamento com adição de lodo, o que pode estar relacionado à decomposição do material orgânico e liberação dos nutrientes (Quadro 4).

Os maiores valores do peso da matéria seca e fresca da parte aérea e da matéria seca das raízes foram observados para o Jatobá-do-cerrado nos tratamento com lodo em associação com os demais resíduos orgânicos (Quadro 3), porém para o peso da matéria seca das raízes esses tratamentos não diferiram do controle nem do tratamento com adição de torta de filtro. Para o Monjoleiro, os tratamentos com lodo ou lodo associado à torta de filtro foram os que proporcionaram maior peso da matéria seca e fresca da parte aérea, enquanto a mistura lodo/torta proporcionou maior peso da matéria seca e fresca das raízes. O Jatobá-do-cerrado e o Monjoleiro exibiram correlações significativas e negativas entre peso da matéria seca da parte aérea e das raízes em relação ao $\mathrm{pH}$ e apresentaram correlação positiva do peso seco e fresco da parte aérea com os teores de $\mathrm{P}, \mathrm{K}^{+}, \mathrm{Ca}^{2+}$, $\mathrm{Mg}^{2+}, \mathrm{SB}$ e CTC (Quadros 5 e 6). 
Os maiores comprimentos radiculares do jatobádo-Cerrado foram observados nos tratamentos controle e maravalha/torta, os quais não diferiram do tratamento com lodo e maravalha aplicados isoladamente,

Quadro 4. Altura das plantas aos 30, 60, 90 e 120 dias após o transplantio, para as plantas de jatobá-doCerrado e monjoleiro, nos diferentes substratos. Média de seis repetições

\begin{tabular}{|c|c|c|c|c|}
\hline Tratamento $^{(1)}$ & 30 dias & 60 dias & 90 dias & 120 dias \\
\hline & \multicolumn{4}{|c|}{ Jatobá-do-Cerrado } \\
\hline $\mathrm{T} 1$ & 18,33 & $22,16 \mathrm{c}$ & $23,66 \mathrm{~d}$ & $24,66 \mathrm{~d}$ \\
\hline $\mathrm{T} 2$ & 19,16 & $26,16 \mathrm{~b}$ & $31,16 \mathrm{c}$ & $34,83 \mathrm{c}$ \\
\hline T3 & 17,66 & $25,16 \mathrm{~b}$ & $37,00 \mathrm{~b}$ & $42,83 \mathrm{~b}$ \\
\hline $\mathrm{T} 4$ & 22,16 & $31,83 \mathrm{a}$ & $47,33 \mathrm{a}$ & $57,33 \mathrm{a}$ \\
\hline T5 & 20,00 & $31,16 \mathrm{a}$ & $46,66 \mathrm{a}$ & $51,66 \mathrm{a}$ \\
\hline $\mathrm{T} 6$ & 16,66 & $18,16 \mathrm{c}$ & $19,66 \mathrm{~d}$ & $20,00 \mathrm{~d}$ \\
\hline $\mathrm{T} 7$ & 17,33 & $22,08 \mathrm{c}$ & $30,16 \mathrm{c}$ & $29,50 \mathrm{c}$ \\
\hline $\mathrm{T} 8$ & 16,16 & $18,50 \mathrm{c}$ & $20,83 \mathrm{~d}$ & $23,83 \mathrm{~d}$ \\
\hline Valor de $\mathrm{F}$ & $2,08^{\mathrm{ns}}$ & $9,54^{* *}$ & $27,26^{* *}$ & $29,35^{* *}$ \\
\hline \multirow[t]{2}{*}{ CV (\%) } & 18,06 & 16,85 & 15,90 & 17,46 \\
\hline & \multicolumn{4}{|c|}{ Monjoleiro } \\
\hline $\mathrm{T} 1$ & $10,16 \mathrm{c}$ & $12,33 \mathrm{~b}$ & $12,00 \mathrm{c}$ & $13,00 \mathrm{~d}$ \\
\hline T2 & $19,33 \mathrm{~b}$ & $55,50 \mathrm{a}$ & $122,33 \mathrm{a}$ & $138,66 \mathrm{a}$ \\
\hline T3 & $18,33 \mathrm{~b}$ & 56,16 a & $103,33 \mathrm{~b}$ & $114,66 \mathrm{~b}$ \\
\hline $\mathrm{T} 4$ & $24,50 \mathrm{a}$ & $61,83 \mathrm{a}$ & $93,83 \mathrm{~b}$ & $102,33 \mathrm{~b}$ \\
\hline T5 & $23,00 \mathrm{a}$ & 58,16 a & $99,00 \mathrm{~b}$ & $124,16 \mathrm{a}$ \\
\hline $\mathrm{T} 6$ & $11,83 \mathrm{c}$ & $11,66 \mathrm{~b}$ & $12,33 \mathrm{c}$ & $11,16 \mathrm{~d}$ \\
\hline $\mathrm{T} 7$ & $12,75 \mathrm{c}$ & $15,16 \mathrm{~b}$ & $17,33 \mathrm{c}$ & $26,83 \mathrm{c}$ \\
\hline $\mathrm{T} 8$ & $11,16 \mathrm{c}$ & $9,66 \mathrm{~b}$ & $20,50 \mathrm{c}$ & $28,66 \mathrm{c}$ \\
\hline Valor de F & $13,49^{* *}$ & $38,79^{* *}$ & $151,90^{* *}$ & $94,62^{* *}$ \\
\hline CV (\%) & 22,87 & 27,54 & 16,0 & 19,71 \\
\hline
\end{tabular}

${ }^{(1)}$ Tratamentos (T) consistiram de: 1. subsolo exposto + inóculo + calcário (controle); 2. controle + lodo de esgoto (L); 3. controle $+\mathrm{L}+$ torta de filtro $(\mathrm{T}) ; 4$. controle $+\mathrm{L}+$ maravalha de madeira $(\mathrm{M}) ; 5$. controle $+\mathrm{L}+\mathrm{T}+\mathrm{M}$; 6 . controle $+\mathrm{M} ; 7$. controle $+\mathrm{T}$; e 8 . controle $+\mathrm{T}+\mathrm{M}$. Médias seguidas de mesma letra, na coluna, não diferem entre si pelo teste de Scott-Knott a $5 \%$. ${ }^{(2)} \mathrm{CV}$ : coeficiente de variação (\%).

Quadro 5. Coeficientes de correlação linear simples entre as característicsa estudadas para o jatobá-doCerrado

\begin{tabular}{|c|c|c|c|c|c|c|c|c|c|c|c|c|c|c|c|c|c|c|}
\hline Variável & COL & $\mathrm{C}-\mathrm{CO}_{2}$ & Altura & MSPA & MFPA & MSSR & MFSR & CSR & $\mathbf{P}$ & MO & $\mathbf{p H}$ & $\mathbf{K}$ & $\mathrm{Ca}$ & Mg & $\mathrm{H}+\mathrm{Al}$ & SB & CTC & V\% \\
\hline Esporos & $\mathrm{ns}$ & ns & $0,37^{*}$ & ns & ns & $0,36^{*}$ & $0,33^{*}$ & ns & $\mathrm{ns}$ & ns & ns & ns & $\mathrm{ns}$ & $\mathrm{ns}$ & ns & $\mathrm{ns}$ & ns & $-0,37^{*}$ \\
\hline COL & - & ns & $\mathrm{ns}$ & $\mathrm{ns}$ & $\mathrm{ns}$ & $0,37^{*}$ & $0,38^{* *}$ & ns & $-0,43^{*}$ & $-0,44^{*}$ & $\mathrm{~ns}$ & $-0,46^{*}$ & $-0,45^{*}$ & $-0,37^{*}$ & $\mathrm{~ns}$ & $-0,44^{*}$ & $\mathrm{~ns}$ & $-0,47^{\text {** }}$ \\
\hline $\mathrm{C}-\mathrm{CO}_{2}$ & - & - & $0,57^{* *}$ & $0,63^{* *}$ & $0,67^{\text {** }}$ & $\mathrm{ns}$ & $0,36^{*}$ & $\mathrm{~ns}$ & $0,49^{*}$ & $0,73^{* *}$ & $-0,76^{* *}$ & $0,45^{*}$ & $\mathrm{~ns}$ & ns & $0,74^{* *}$ & ns & $0,44^{*}$ & $-0,67^{* *}$ \\
\hline Altura & - & - & - & $0,82^{* *}$ & $0,80^{* *}$ & $0,56^{* *}$ & $0,66^{* *}$ & $\mathrm{~ns}$ & $0,64^{* *}$ & * ns & $-0,60^{* *}$ & $0,53^{*}$ & $\mathrm{~ns}$ & $0,42^{*}$ & $0,69^{* *}$ & $0,39^{*}$ & $0,57^{* *}$ & $-0,47^{* *}$ \\
\hline MSPA & - & - & - & - & $0,96^{\star *}$ & $0,52^{* *}$ & $0,61^{\text {** }}$ & $-0,32^{*}$ & $0,64^{* *}$ & $\mathrm{~ns}$ & $-0,64^{* *}$ & $0,47^{\star}$ & $0,37^{*}$ & $0,41^{*}$ & $0,72^{* *}$ & $0,40^{*}$ & $0,59^{* *}$ & $-0,47^{k * k}$ \\
\hline MFPA & - & - & - & - & - & $0,48^{* *}$ & $0,62^{* *}$ & $-0,40^{* *}$ & $0,66^{* *}$ & $\mathrm{~ns}$ & $-0,68^{* *}$ & $0,48^{*}$ & $0,39^{*}$ & $0,44^{*}$ & $0,78^{\star * *}$ & $0,43^{*}$ & $0,64^{* *}$ & $-0,51^{* * *}$ \\
\hline MSSR & - & - & - & - & - & - & $0,90^{* *}$ & $\mathrm{~ns}$ & ns & ns & ns & $\mathrm{ns}$ & $\mathrm{ns}$ & ns & $0,41^{*}$ & ns & $\mathrm{ns}$ & $-0,44^{*}$ \\
\hline MFSR & - & - & - & - & - & - & - & $-0,35^{*}$ & ns & ns & $-0,37^{*}$ & ns & ns & ns & $0,54^{* *}$ & ns & ns & $-0,51^{* *}$ \\
\hline CSR & - & - & - & - & - & - & - & - & $\mathrm{ns}$ & ns & $\mathrm{ns}$ & $\mathrm{ns}$ & $\mathrm{ns}$ & $\mathrm{ns}$ & $\mathrm{ns}$ & $\mathrm{ns}$ & $\mathrm{ns}$ & $\mathrm{ns}$ \\
\hline $\mathrm{P}$ & - & - & - & - & - & - & - & - & - & $\mathrm{ns}$ & $-0,65^{* k}$ & $0,92^{* *}$ & $0,80^{* *}$ & ${ }^{k} 0,84^{* * *}$ & $0,63^{* *}$ & $0,86^{* *}$ & $0,91^{* *}$ & $\mathrm{~ns}$ \\
\hline MO & - & - & - & - & - & - & - & - & - & - & $-0,59^{* *}$ & $\mathrm{~ns}$ & ns & ns & $0,57^{* k}$ & $\mathrm{~ns}$ & $\mathrm{~ns}$ & $-0,76^{* * *}$ \\
\hline $\mathrm{pH}$ & - & - & - & - & - & - & - & - & - & - & $-\quad$. & $-0,64^{* *}$ & ns & $-0,47^{* *}$ & $-0,90^{* *}$ & $-0,40^{*}$ & $-0,63^{* *}$ & $0,69^{* * *}$ \\
\hline $\mathrm{K}$ & - & - & - & - & - & - & - & - & - & - & - & - & $0,78^{* *}$ & ${ }^{*} 0,93^{* *}$ & $\mathrm{~ns}$ & $0,98^{* *}$ & $0,88^{* *}$ & $\mathrm{~ns}$ \\
\hline $\mathrm{Ca}$ & - & - & - & - & - & - & - & - & - & - & - & - & - & $0,93^{\star *}$ & $0,47^{*}$ & $0,98^{*}$ & $0,88^{* *}$ & ns \\
\hline $\mathrm{Mg}$ & - & - & - & - & - & - & - & - & - & - & - & - & - & - & $0,47^{*}$ & $0,41^{*}$ & $0,70^{* *}$ & $-0,78^{* * *}$ \\
\hline $\mathrm{H}+\mathrm{Al}$ & - & - & - & - & - & - & - & - & - & - & - & - & - & - & - & $0,41^{*}$ & $0,70^{* *}$ & $-0,78^{* * *}$ \\
\hline SB & - & - & - & - & - & - & - & - & - & - & - & - & - & - & - & - & $0,93^{* *}$ & $\mathrm{~ns}$ \\
\hline CTC & - & - & - & - & - & - & - & - & - & - & - & - & - & - & - & - & - & $\mathrm{ns}$ \\
\hline
\end{tabular}

* $\mathrm{e}^{* *}$ : significativo a 5 e $1 \%$, respectivamente; (ns) não significativo. COL: colonização micorrízica, C-CO ${ }_{2}$ : atividade respiratória microbiana, MSPA: peso da matéria seca da parte aérea, MFPA: peso da matéria fresca da parte aérea, MSSR: peso da massa seca do sistema radicular, MFSR: peso da matéria fresca do sistema radicular, CSR: comprimento do sistema radicular, P: fósforo, MO: matéria orgânica, K: potássio, Ca: cálcio, Mg: magnésio, Al: alumínio, CTC: capacidade de troca catiônica e V (\%): saturação por bases. 
Quadro 6. Coeficientes de correlação linear simples entre as características estudadas para o monjoleiro

\begin{tabular}{|c|c|c|c|c|c|c|c|c|c|c|c|c|c|c|c|c|c|c|}
\hline Variável & COL C & $\mathrm{C}-\mathrm{CO}_{2}$ & Altura & MSPA & MFPA & MSSR & MFSR & CSR & $\mathbf{P}$ & MO & pH & $\mathbf{K}$ & $\mathbf{C a}$ & Mg & $\mathrm{H}+\mathbf{A l}$ & SB & CTC & V\% \\
\hline Esporos & $0,48^{* *}$ & ns & $\mathrm{ns}$ & $0,38^{* *}$ & $0,34^{*}$ & $\mathrm{~ns}$ & $\mathrm{~ns}$ & $\mathrm{~ns}$ & $0,42^{*}$ & $\mathrm{~ns}$ & $\mathrm{~ns}$ & $\mathrm{~ns}$ & $\mathrm{~ns}$ & $\mathrm{~ns}$ & $\mathrm{~ns}$ & $0,36^{*}$ & $0,42^{*}$ & ns \\
\hline COL & - & $0,48^{* *}$ & $0,66^{* *}$ & $0,58^{* *}$ & $0,59^{* *}$ & $0,39^{* *}$ & $0,46^{* *}$ & $\mathrm{~ns}$ & $0,50^{* *}$ & $\mathrm{~ns}$ & $-0,49^{*}$ & $0,54^{* *}$ & $\mathrm{~ns}$ & $0,36^{*}$ & $0,48^{* *}$ & $0,36^{*}$ & $0,47^{\text {** }}$ & $\mathrm{ns}$ \\
\hline $\mathrm{C}-\mathrm{CO}_{2}$ & - & - & $0,77^{\text {** }}$ & $0,68^{* *}$ & $0,69^{* *}$ & $0,64^{* *}$ & $0,66^{* *}$ & $0,28^{*}$ & $0,72^{* *}$ & $0,57^{* *}$ & $-0,64^{* *}$ & $0,75^{\star *}$ & $0,58^{* *}$ & $0,59^{* *}$ & $0,59^{* *}$ & $0,64^{* *}$ & $0,74^{* *}$ & $\mathrm{~ns}$ \\
\hline Altura & - & - & - & $0,89^{* \star}$ & $0,92^{* *}$ & $0,83^{k * k}$ & $0,85^{\text {** }}$ & $\mathrm{ns}$ & $0,89^{* *}$ & $\mathrm{~ns}$ & $-0,67^{* *}$ & $0,81^{* * k}$ & $0,48^{* *}$ & $0,59^{* *}$ & $0,68^{\text {** }}$ & $0,60^{* k}$ & $0,74^{\star *}$ & $\mathrm{~ns}$ \\
\hline MSPA & - & - & - & - & $0,99^{* *}$ & $0,93^{\star *}$ & $0,98^{* *}$ & $\mathrm{~ns}$ & $0,92^{* *}$ & $\mathrm{~ns}$ & $-0,59^{* *}$ & $0,73^{* *}$ & $0,55^{\text {** }}$ & $0,66^{* *}$ & $0,64^{* *}$ & $0,67^{\text {** }}$ & $0,78^{* *}$ & $\mathrm{~ns}$ \\
\hline MFPA & - & - & - & - & - & $0,93^{\text {** }}$ & $0,92^{* *}$ & $\mathrm{~ns}$ & $0,93^{* *}$ & $\mathrm{~ns}$ & $-0,60^{* *}$ & $0,75^{\text {** }}$ & $0,55^{\text {** }}$ & $0,66^{* *}$ & $0,64^{* *}$ & $0,67^{\text {** }}$ & $0,78^{* *}$ & ns \\
\hline MSSR & - & - & - & - & - & - & $0,98^{* * *}$ & $0,28^{*}$ & $0,87^{\text {** }}$ & ns & $-0,65^{\text {** }}$ & $0,63^{\text {** }}$ & $0,43^{* *}$ & $0,57^{\text {** }}$ & $0,72^{* *}$ & $0,55^{\text {** }}$ & $0,71^{* *}$ & ns \\
\hline MFSR & . & . & - & - & - & - & - & $0,29^{*}$ & $0,84^{\text {** }}$ & $\mathrm{ns}$ & $-0,73^{* *}$ & $0,59^{\text {** }}$ & $0,43^{*}$ & $0,53^{\text {** }}$ & $0,79^{* *}$ & $0,53^{* *}$ & $0,72^{\star *}$ & ns \\
\hline CSR & & - & - & - & - & - & - & - & $\mathrm{ns}$ & $\mathrm{ns}$ & $\mathrm{ns}$ & $\mathrm{ns}$ & $\mathrm{ns}$ & $\mathrm{ns}$ & $\mathrm{ns}$ & $\mathrm{ns}$ & $\mathrm{ns}$ & ns \\
\hline $\mathrm{P}$ & & - & - & - & - & - & - & - & - & $\mathrm{ns}$ & $-0,49^{* *}$ & $0,83^{* *}$ & $0,57^{* *}$ & $0,71^{* *}$ & $0,55^{* *}$ & $0,70^{* *}$ & $0,78^{* *}$ & $\mathrm{~ns}$ \\
\hline MO & - & - & - & - & - & - & - & - & - & - & $-0,77^{* *}$ & $\mathrm{~ns}$ & $\mathrm{~ns}$ & $\mathrm{~ns}$ & $0,65^{\text {** }}$ & $\mathrm{ns}$ & $\mathrm{ns}$ & $-0,58^{* *}$ \\
\hline $\mathrm{pH}$ & - & - & - & - & - & - & - & - & - & - & - & $-0,45^{*}$ & $\mathrm{~ns}$ & ns & $-0,90^{* *}$ & $\mathrm{~ns}$ & $-0,59^{* *}$ & $0,70^{* *}$ \\
\hline $\mathrm{K}$ & & - & - & - & - & - & - & - & - & - & - & - & $\mathrm{ns}$ & $0,55^{\star * *}$ & * $\mathrm{ns}$ & $0,53^{* *}$ & $0,59^{* *}$ & ns \\
\hline $\mathrm{Ca}$ & - & . & - & - & - & - & - & - & - & - & - & - & - & $0,71^{\text {** }}$ & $\mathrm{ns}$ & $0,93^{* *}$ & $0,86^{\text {** }}$ & $0,42^{*}$ \\
\hline $\mathrm{Mg}$ & . & . & - & - & - & - & - & - & - & - & - & - & - & - & $\mathrm{ns}$ & $0,90^{* *}$ & $0,87^{\text {** }}$ & $0,37^{*}$ \\
\hline $\mathrm{H}+\mathrm{Al}$ & - & - & - & - & - & - & - & - & - & - & - & - & - & - & - & $\mathrm{ns}$ & $0,63^{* *}$ & $-0,71^{\text {** }}$ \\
\hline SB & - & - & - & - & - & - & - & - & - & - & - & - & - & - & - & - & $0,93^{* *}$ & $0,41^{*}$ \\
\hline CTC & - & - & - & - & - & - & - & - & - & - & - & - & - & - & - & - & - & $\mathrm{ns}$ \\
\hline
\end{tabular}

* $\mathrm{e}^{* *}$ : significativo a 5 e $1 \%$, respectivamente; (ns) não significativo. COL: colonização micorrízica, C-CO ${ }_{2}$ atividade respiratória microbiana, MSPA: peso da matéria seca da parte aérea, MFPA: peso da matéria fresca da parte aérea, MSSR: peso da massa seca do sistema radicular, MFSR: peso da matéria fresca do sistema radicular, CSR: comprimento do sistema radicular, P: fósforo, MO: matéria orgânica, K: potássio, Ca: cálcio, Mg: magnésio, Al: alumínio, CTC: capacidade de troca catiônica e V (\%): saturação por bases.

enquanto para o monjoleiro apenas lodo e maravalha e torta de filtro diferiram do controle, com os menores valores (Quadro 3). As duas espécies não mostraram correlações significativas entre comprimento radicular e características químicas do solo, porém o jatobá-doCerrado exibiu correlações negativas entre o peso da matéria seca das raízes e o comprimento radicular da matéria seca e fresca da parte aérea, enquanto o monjoleiro mostrou correlações positivas com relação à matéria fresca e seca das raízes (Quadros 5 e 6).

A adição de resíduos orgânicos, juntamente com o lodo de esgoto, elevou os teores de $\mathrm{P}$ e não exerceu efeito negativo sobre a colonização micorrízica (Quadro 3), o que está relacionado com um maior desenvolvimento das plantas em resposta à elevação da fertilidade (Faria et al., 1995). O sucesso do crescimento e do estabelecimento das leguminosas arbóreas em solos degradados e de baixa fertilidade natural é, geralmente, limitado pela elevada acidez e pela deficiência acentuada de nutrientes (Malajczuk et al., 1993).

As variáveis microbiológicas também responderam positivamente à adição do lodo de esgoto isoladamente ou associado aos demais resíduos orgânicos. Nas duas espécies arbóreas (Quadro 3), verificou-se menor atividade microbiana no tratamento controle e maior atividade no tratamento com a mistura lodo/torta/ maravalha. As duas espécies apresentaram correlação negativa entre $\mathrm{C}-\mathrm{CO}_{2}$ liberado e $\mathrm{pH}$ (Quadros 3 e 4). As maiores atividades microbianas foram detectadas nos tratamentos que receberam lodo ou lodo adicionado dos demais resíduos orgânicos, possivelmente por serem fontes de nutrientes (especialmente de $\mathrm{P}$ ) e de matéria orgânica (Quadro 1), fundamentais para a atividade microbiana (Quadro 3). Assim como no presente trabalho, Vargas \& Scholles (2000) relatam o aumento da atividade microbiana, avaliada pelo $\mathrm{C}$ $\mathrm{CO}_{2}$ liberado, justificado pelo acúmulo de matéria orgânica.

O tratamento com adição de torta/maravalha, por sua vez, apresentou os menores teores de $\mathrm{P}$ e matéria orgânica (Quadro 2) e, nesse caso, não favoreceu a atividade microbiana. De fato, comparado à maravalha e à torta de filtro, o lodo de esgoto contém componentes de mais rápida decomposição microbiana. Assim como verificado no presente trabalho, Vargas \& Scholles (2000) relatam que os valores de $\mathrm{C}-\mathrm{CO}_{2}$ liberados foram superiores nos tratamentos com adição de lodo de esgoto, quando comparados aos da testemunha e do tratamento com fertilizante mineral. Esses autores acrescentam que essa variável constitui um indicativo de qualidade adequado ao monitoramento da melhoria de uma área em recuperação para um curto período de observação.

A maioria das espécies de plantas apresenta-se colonizada por FMA, os quais têm potencial para absorver nutrientes, destacando sua importância para os solos tropicais com alta capacidade de fixação de fosfatos e baixos teores de nutrientes disponíveis (Moreira \& Siqueira, 2006). No presente trabalho, para o jatobá-do-Cerrado (Quadro 3), os tratamentos com adição de resíduos orgânicos, na presença ou ausência do lodo, mostraram as mais altas percentagens de colonização micorrízica e diferiram do controle. Entretanto, para o monjoleiro, as maiores percentagens de colonização foram observadas nos tratamentos com adição de lodo e com lodo/torta/ maravalha, os quais também diferiram do controle, que apresentou valores menores para colonização. $\mathrm{O}$ tipo do substrato influenciou a simbiose, possivelmente 
pela quantidade de nutrientes disponíveis, como comentado por Silva et al. (2004). A falta de correlação entre crescimento de raízes e colonização observada no presente trabalho pode ser explicada pelo fato de que, em leguminosas arbóreas, a presença de micorriza proporciona expansão do sistema radicular para absorção de P, Mo, Zn e outros nutrientes de baixa mobilidade no solo, que chegam até as raízes pelo processo de difusão, permitindo o crescimento em solos extremamente pobres, como o subsolo empregado neste trabalho (Caldeira et al., 1999).

Vários autores têm mostrado que a alta disponibilidade de $\mathrm{P}$ no solo promove restrição à infecção micorrízica e redução da percentagem de raízes colonizadas (Melloni et al., 2000). Neste trabalho, as duas espécies de plantas exibiram comportamentos semelhantes diante das diferentes variáveis analisadas. As maiores médias de crescimento, para a maioria das variáveis, foram verificadas nos tratamentos com lodo ou com lodo + resíduos, onde foram detectados, também, os maiores teores de $\mathrm{P}$ no substrato. Entretanto, a colonização micorrízica e o teor de $\mathrm{P}$ exibiram correlações positiva para o jatobádo-Cerrado e negativa para o monjoleiro (Quadros 5 e 6 ).

Em áreas degradadas, os esporos de FMA podem ser a fonte mais importante de propágulos, devido à sua resistência aos estresses mais severos (Brundrett et al., 1991). A capacidade de esporulação dos FMA pode variar com a planta hospedeira, assim como em função das características edáficas (Douds, 1994). As duas espécies exibiram, para número de esporos, os maiores valores nos tratamentos com adição de lodo/ maravalha e lodo/torta/maravalha, além do tratamento com torta de filtro para jatobá-do-Cerrado e do tratamento com lodo para o monjoleiro (Quadro 3). Nos tratamentos lodo/maravalha e lodo/torta/ maravalha pode ser verificado aumento aproximado na esporulação, em relação ao controle, de 10 e 5 vezes, respectivamente, para o jatobá-do-Cerrado e de 7 e 17 vezes, respectivamente, para o monjoleiro. Correlação positiva foi verificada entre esporos e altura, massa seca da parte aérea, massa de matéria seca das raízes e massa fresca da parte aérea para o jatobá-do-Cerrado, e com colonização, massa de matéria seca e fresca da parte aérea, P e CTC, para monjoleiro (Quadros 5 e $6)$.

Com base nos resultados, pode-se verificar a eficácia do lodo como fonte de nutrientes para o subsolo. Considerando o efeito residual, ou seja, um período mais longo de decomposição dos resíduos mais ricos em C, como a maravalha de madeira e a torta de filtro, seria interessante combiná-los com lodo de esgoto, resíduo de alta relação $\mathrm{C}: \mathrm{N}$ e rico em nutrientes. Isso aceleraria a decomposição e, consequentemente, melhoraria as propriedades químicas, físicas e microbiológicas do solo, tornando uma alternativa viável para o crescimento das plantas e, dessa forma, possibilitando a recuperação do subsolo exposto.

\section{CONCLUSÕES}

1. As misturas contendo lodo de esgoto associado a torta de filtro e maravalha de madeira foram as que promoveram os maiores incrementos na qualidade do subsolo, com perspectivas de sua recuperação.

2. Os tratamentos com lodo de esgoto proporcionaram maior atividade microbiana no solo e maior crescimento para as duas espécies arbóreas.

3. A presença do lodo de esgoto promoveu melhora na fertilidade do solo, com aumento dos teores de $\mathrm{P}$, $\mathrm{MO}, \mathrm{K}^{+}, \mathrm{Ca}^{2+} \mathrm{e} \mathrm{Mg}^{2+}$. A matéria orgânica e o $\mathrm{P}$ tiveram seus teores elevados nos tratamentos com mistura de lodo de esgoto e demais resíduos.

4. O maior crescimento vegetativo das duas espécies arbóreas estudadas foi proporcionado pelo uso isolado do lodo de esgoto ou em mistura com os demais resíduos.

5. A atividade microbiana e a colonização micorrízica mostraram-se mais elevadas nos tratamentos que receberam lodo de esgoto ou lodo misturado com resíduos.

\section{LITERATURA CITADA}

ANDERSON, T.H. \& DOMSCH, K.H. Ratio of microbial biomass carbon to total organic carbon inarable soils. Soil Biol. Biochem., 21:471-479, 1982.

BRANCO, C.M.; BUDZIAK, C.R.; PAIXÃO, R.E. \& MANGRICH, A.S. Aproveitamento de resíduo florestal para húmus. R. Madeira, 89, 2005. Embrapa Floresta. Disponível em: <http://www.remade.com.br/revista/ materia.php?edicao=89\&id=738> Acesso em abril de 2006 .

BRUNDRETT, M.; BOUGHER, N.; DELL, B.; GRAVE, T. \& MALAJCZUK, N. Working with mycorrhiza in forestry and agriculture. Canberra, Australian Centre for International Agricultural Research, 1996. 374p. (Monograph, 32).

CALDEIRA, M.V.W.; SILVCA, E.M.R.; FRANCO, A.A. \& ZANON, M.L.B. Crescimento de leguminosas arbóreas em respostas a inoculação com fungos micorrízicos arbusculares. Ci. Flor., 7:1-10, 1997.

CALDEIRA, M.V.W.; SILVCA, E.M.R.; FRANCO, A.A. \& ZANON, M.L.B. Efeito de fungos micorrízicos arbusculares no desenvolvimento de duas leguminosas arbóreas. Ci. Flor., 9:63-70, 1999.

CATAN, R.A. \& ALONSO, O. Avaliação da exigência de calcário do solo. An. ESALQ, 26:141-156, 1969.

COMPANHIA DE SANEAMENTO BÁSICO DO ESTADO DE SÃO PAULO - CETESB. Aplicação de lodos de sistemas de tratamentos biológicos em áreas agrícolas: Critérios para projeto e operação. São Paulo, 1999. 32p.

COLODRO, G.; ESPÍNDOLA, C.R.; CASSIOLATO, A.M.R. \& ALVES, M.C. Atividade microbiana em um Latossolo degradado tratado com lodo de esgoto. Agriambi, 11:195$198,2007$. 
DEMATTÊ, J.L.I. Levantamento detalhado dos solos do campus experimental de Ilha Solteira, Piracicaba, ESALQ/ USP, 1980. 114p.

DOUDS, D.D. Relationships between hyphal and arbuscular colonization and sporulation in mycorrhiza of Paspalum notatum. New Phytol., 126:233-237, 1994.

DUDA, G.P.; CAMPELLO, E.F.C.; MENDONÇA, E.S.; LOURES, J.L. \& DOMINGOS, M. Avaliação de frações da matéria orgânica do solo para caracterização de áreas degradadas. R. Bras. Ci. Solo, 23:723-728, 1999.

FARIA, M.P.; VALE, F.R.; SIQUEIRA, J.O. \& CURI, N. Crescimento de leguminosas arbóreas em resposta a fósforo, nitrogênio, fungos micorrízicos e rizóbio. II. Peltophorum dublim (Spreng.) TAUB. R. Árvore, 19:433466, 1995.

FERNANDES, C. \& MURAOKA, T. Absorção de P por híbridos de milho cultivados em solo de Cerrado. Sci. Agr., 59:781787, 2002.

GERDEMANN, J.W. \& NICOLSON, T.H. Spores of micorrhizal endogone species extracted from soil by wet sieving and decanting. Trans. Br. Mycol. Soc., 46:234-244, 1963.

JENKINS, W.R. A rapid centrifugal-flotation technique for separating nematodes from soil. Plant Soil, 73:288-300, 1964.

MALAJCZUK, N.; JONES, N. \& NEELY, C. The importance of mycorrhiza to forest trees. Washington, The World Bank, Asia Technical Department, 1993. 27p. (Land Resources Series, 2)

MELLONI, R.; NOGUEIRA, M.A.; FREIRE, V.F. \& CARDOSO, E.J.B.N. P adicionado e fungos micorrízicos arbusculares no crescimento e nutrição mineral de limoeiro-cravo (Citrus limonia (L.) Osbeck.). R. Bras. Ci. Solo, 24:767$775,2000$.

MOREIRA, F.M.S. \& SIQUEIRA, J.O. Microbiologia e bioquímica do solo. Lavras, Universidade Federal de Lavras, 2006. p.543-662.

M. NETO, F.C. \& SOUZA, C.M. Avaliação de um método de recuperação de áreas degradadas através da estimação da respiração da microbiota. In: REUNIÃO BRASILEIRA DE MANEJO E CONSERVAÇÃO DO SOLO E DA ÁGUA, 13., 2000, Ilhéus. Anais. Ilhéus, Sociedade Brasileira de Ciência do Solo, 2000. CD ROM.
PHILLIPS, J.M. \& HAYMAN, D.S. Improved procedures for clearing roots and staining parasitic and vesiculararbuscular mycorrhizal fungi for rapid assessment of infection. Trans. Br. Myol. Soc., 55:158-161, 1970.

PRASAD, M. Response of sugarcane to filter press mud and $\mathrm{N}, \mathrm{P}$ and $\mathrm{K}$ fertilizers. I. Effect on sugarcane yield and sucrose content. Agron. J., 68:539-543, 1976.

RAIJ, B.van \& QUAGGIO, J.A. Métodos de análises de solos para fins de fertilidade. Campinas, Instituto Agronômico de Campinas, 1983. 31p. (Boletim Técnico, 81).

RAIJ, B.van; CANTARELLA, H.; QUAGGIO J.A. \& FURLANI, A.M.C. Recomendações de adubação e calagem para o Estado de São Paulo. 2.ed. Campinas, Instituto Agronômico de Campinas, 1997. 285p. (Boletim Técnico, 100)

RODRIGUES, G.B.; MALTONI, K.L. \& CASSIOLATO, A.M.R. Dinâmica da regeneração do subsolo de áreas degradadas dentro do bioma Cerrado. Agriambi, 11:73-80, 2007.

SANTOS, A.C.; SILVA, I.F.; LIMA, J.R.S.; ANDRADE, A.P. \& CAVALCANTE, V.R. Gramíneas e leguminosas na recuperação de áreas degradadas: Efeito nas características químicas de solo. R. Bras. Ci. Solo, 25:10631071, 2001.

SEVERINO, L.S.; COSTA, F.X.; BELTRÃO, N.E.M.; LUCENA, M.A. \& GUIMARÃES, M.M.B. Mineralização da torta de mamona, esterco bovino e bagaço de cana estimada pela respiração microbiana. R. Biol. Ci. Terra, 5:2004. Disponível em: <http://www.uepb.edu.br/eduep/rbct/sumarios/pdf/ esterco.pdf $>$. Acesso em 24 de abril de 2007.

SILVA, M.A.; CAVALCANTE, T.; SILVA, F.S.B.; SOARES, S.A.G. \& MAIA, L.C. Crescimento de mudas de maracujazeiro-doce (Passiflora alata Curtis) associadas a fungos micorrízicos arbusculares (Glomeromycota). Acta Bot. Bras., 18:981-985, 2004.

TSUTIYA, M.T. Características de biossólidos gerados em estações de tratamento de esgoto. In: TSUTIYA, M.T.; COMPARINI, J.B.; ALEM SOBRINHO, P.; HESPANHOL, I.; CARVAlHO, P.C.T.; MELFI, A.J.; MELO, W.J. \& MARQUES, M.O., eds. Biossólidos na agricultura. São Paulo, SABESP, 2001. p.89-131.

UNITED STATES ENVIRONMENT PROTECTION AGENCY - USEPA. Standart for the use or disposal of sewage sludge. Fed. Reg., 53:9248-9415, 1993.

VARGAS, L.K. \& SCHOLLES, D. Biomassa microbiana e produção de $\mathrm{C}-\mathrm{CO}_{2}$ e $\mathrm{N}$ mineral em um Podzólico Vermelho-Escuro submetido a diferentes sistemas de manejo. R. Bras. Ci. Solo, 24:35-42, 2000. 